\title{
PRIMITIVE IDEALS OF TWISTED GROUP ALGEBRAS
}

\author{
BY
}

\author{
OTHA L. BRITTON
}

\begin{abstract}
E. Effros and F. Hahn have conjectured that if $(G, Z)$ is a second countable locally compact transformation group, with $G$ amenable, then every primitive ideal of the associated $C^{*}$-algebra arises as the kernel of an irreducible representation induced from a stability subgroup. Results of Effros and Hahn concerning this conjecture are extended to include the twisted group algebra $L^{1}(G, A ; T, \alpha)$, where $A$ is a separable type I $C^{*}$-algebra.
\end{abstract}

1. Introduction. Twisted group algebras in their most general setting were introduced by Horst Leptin in [11], [12], and [13] (under the name of generalized group algebras) and independently by R. C. Busby and H. A. Smith in [4]. Included as special cases of twisted group algebras are the usual group algebras $\left(L^{1}(G)\right.$ equipped with convolution and involution), transformation group algebras, covariance algebras (which are studied in quantum physics) and the group algebras of group extensions. One of the most important concepts in the theory of twisted group algebras is that of induced representation. In [14], G. W. Mackey defined this concept for the theory of unitary representations of locally compact groups and studied the problem of trying to obtain all (up to equivalence) unitary representations of a group by inducing from certain subgroups. Mackey showed that when a particular condition (which he called "regularly imbedded") is satisfied, one may obtain all such representations in this manner, but that if this condition is not satisfied, one cannot in general obtain all such representations. In [17], M. Takesaki generalized this result to the case of covariance algebras, and in [10], H. Leptin generalized this result to the case of twisted group algebras, but where the element $T$ in the twisting pair $(T, \alpha)$ (see $\S 2)$ is multiplicative.

In the setting of transformation groups, E. Effros and F. Hahn conjectured in [6] that even though it may be impossible to obtain all representations by inducing (from the desired subgroups), possibly one may at least obtain the primitive ideal space in this manner. Effros and Hahn obtained some results along this

Presented to the Society, January 25, 1973; received by the editors November 19, 1973 and, in revised form, March 5, 1974.

AMS (MOS) subject classifications (1970). Primary 46L05, 46L25; Secondary 22D15, 22D20, 22D30, 22D45.

Key words and phrases. Twisted group algebra, irreducible representation, induced representation, covariance algebra, group extension, transformation group, dual space. 
line, and we give a generalization of these results to the case of twisted group algebras.

In $\S 2$, we give various definitions and other preliminary material needed in the paper. The induced representations defined by Busby and Smith in [4] are not general enough for the problem studied here, so in $\$ 3$ we define an induced representation which follows more closely the definition given by Takesaki in [17] (and which reduces to it for covariance algebras). For rather special circumstances, we also give a definition of induced representation along the lines of Effros and Hahn in [6], and we show that under these circumstances, the two definitions give rise to equivalent representations. In [10], $\mathrm{H}$. Leptin does much of the work that we present in the first part of $\S 3$, but he does not include the completely general case. (In particular, Leptin's work does not include all group extensions.) He also uses different notations and constructions throughout his work.

In $\S 4$, we obtain some results which are needed in proving the major theorems of the paper. Included in $\$ 4$ is a result concerning the problem of obtaining the dual space of the twisted group algebra. It is an extension of results presented by Takesaki in [17] and Leptin in [10] .

The main results are presented in $\S 5$. The concern here is the conjecture made by Effros and Hahn in [6]. The problem is to obtain the primitive ideal space of the twisted group algebra and actually consists of two parts-kernel containment in both directions. We present results for both parts of the problem.

In $\$ 6$ we make some concluding remarks regarding possibilities of extending the results presented here.

This paper forms a portion of the author's doctoral dissertation written at Drexel University under the direction of Professor Robert C. Busby.

2. Definitions and preliminary remarks. In the discussions and proofs in this paper, we will need a number of definitions, theorems, and basic facts from the literature, and we review some of these in this section. We first note that throughout this paper, group will always mean locally compact second countable group, and the algebra $A$ will always be a separable $C^{*}$-algebra.

Let $G$ be a group. The mapping $x \rightarrow U(x)$ from $G$ into the unitary operators on a separable Hilbert space $H$ is said to be a continuous unitary representation of $G$ if $U(x y)=U(x) U(y)$ for every $x, y \in G$ and if for every $v \in H$,

$$
\left\|U(x) v-U\left(x_{0}\right) v\right\| \rightarrow 0 \text { as } x \rightarrow x_{0} .
$$

(We could replace the latter condition by weak measurability. See [16, Corollary, p. 279].) We will usually drop the adjective "continuous". By a representation 
$\pi$ of a Banach *-algebra $C$ on $H$, we mean a homomorphism of $C$ into $B(H)$ (the space of bounded linear operators on $H$ ) satisfying $\pi\left(a^{*}\right)=\pi(a)^{*}$ and such that the linear span of $\pi(C) H$ is dense in $H$. The commutant of a set $F$ of operators in $B(H)$, denoted $F^{\prime}$, is the set of elements in $B(H)$ which commute with every element of $F$. If $\pi$ is a representation of $C$ on $H, \pi$ is irreducible if $\pi(C)^{\prime}$ consists only of multiples of the identity operator on $H$. Two representations $\pi_{1}$ on $H_{1}$ and $\pi_{2}$ on $H_{2}$ are equivalent if there exists a unitary $V$ mapping $H_{1}$ onto $H_{2}$ such that $V \pi_{1}(a)=\pi_{2}(a) V$ for all $a \in C$. Similar definitions pertain to unitary representations of $G$. The dual space $\hat{C}$ of $C$ is the space of equivalence classes of irreducible representations of $C$, equipped with the quotient topology (see $[5, \S 3.5]$ ), and the primitive ideal space, Prim $C$, is the space of kernels of irreducible representations, equipped with the hull-kernel topology. (See [5, §3.1].)

Let $A$ be, as usual, a separable $C^{*}$-algebra. A double centralizer on $A$ is a pair $\left(m_{1}, m_{2}\right)$ of bounded linear mappings from $A$ to $A$ such that for all $a, b$ in $A, a\left(m_{1} b\right)=\left(m_{2} a\right) b$. The double centralizers form an algebra $M(A)$ which contains an isomorphic copy of $A$ as a closed two-sided ideal. Representations of $A$ extend uniquely to representations of $M(A)$. For details, see [2] and [9].

We now state the definition of twisted group algebra as given in [4]. Let $d y$ and $\Delta$ be a left Haar measure for $G$ and the corresponding modular function, respectively (see [16, p. 367]). Let $L^{1}(G, A)$ be the Banach space (see [8]) of Bochner-integrable $A$-valued functions on $G$ (that is, functions which are the limit almost everywhere of a sequence of "step functions" and whose norms are integrable), and let $\mathrm{Aut}_{1}(A)$ be the isometric *-automorphisms of $A$ with the strong topology. Let $T$ be a Borel measurable mapping from $G$ to Aut $_{1}(A)$, and let $\alpha$ be a Borel map from $G \times G$ to the unitary double centralizers in $M(A)$ such that

(1) $[T(x) \alpha(y, z)] \alpha(x, y z)=\alpha(x, y) \alpha(x y, z)$,

(2) $[T(x) T(y) a] \alpha(x, y)=\alpha(x, y)[T(x y) a]$,

(3) $\alpha(x, e)=\alpha(e, y)=1, T(e)=1$

for all $x, y, z$ in $G$ and $a$ in $A$, with $e$ being the identity in $G$. Let $p$ : $\operatorname{Aut}_{1}(A) \rightarrow \operatorname{Aut}_{1}(A) / B$ be the natural projection, where $B$ is the subgroup of Aut $_{1}(A)$ consisting of inner automorphisms by unitaries. If $\operatorname{Aut}_{1}(A) / B$ is given the quotient topology, $p$ will be continuous. If $p T: G \rightarrow \operatorname{Aut}_{1}(A) / B$ is continuous, $(T, \alpha)$ is called a twisting pair for $G$ and $A$. We will always assume that $(T, \alpha)$ is a twisting pair. Define a product and an involution, ${ }^{*}$, on $L^{1}(G, A)$ by

$$
(f h)(x)=\int_{G} f(y)\left[T(y) h\left(y^{-1} x\right)\right] \alpha\left(y, y^{-1} x\right) d y
$$


and

$$
f^{*}(x)=\alpha\left(x, x^{-1}\right) *\left[T(x) f\left(x^{-1}\right)^{*}\right] \Delta\left(x^{-1}\right) .
$$

The result is a Banach *-algebra which we denote by $L^{1}(G, A ; T, \alpha)$, the twisted group algebra on $G$ with values in $A$ produced by the twisting pair $(T, \alpha)$. We denote by $C^{*}(G, A ; T, \alpha)$ the enveloping $C^{*}$-algebra of $L^{1}(G, A ; T, \alpha)$ (see $\left.[5, \S 2.7]\right)$.

The proofs of our main results depend rather heavily upon being able to apply the Corollary in [3]. For this, we need the concept of a smooth twisting pair as defined by Busby. A twisting pair $(T, \alpha)$ is said to be smooth if the following two conditions are satisfied:

(1) There is a neighborhood $N$ of the identity $e$ in $G$ such that the restrictions of $T$ and $\alpha$ to $N$ and $N \times N$, respectively, are continuous.

(2) For each $x$ in $G$, there is a neighborhood $N_{x}$ of $e$ in $G$ such that $\beta\left(x^{-1}, y, x\right)$ is continuous in $N_{x}$ as a function of $y$, where $\beta(x, y, z)=$ $\alpha(x, y) \alpha(x y, z)$.

The representations of $L^{1}(G, A ; T, \alpha)$ are in a one-to-one correspondence with pairs of the form $(\pi, U)$, where:

(1) $\pi$ is a representation of $A$ on $H$.

(2) $U$ is a weakly measurable mapping from $G$ to the unitary operators on $H$ such that $U(x) U(y)=\pi(\alpha(x, y)) U(x y)$ for every $x, y$ in $G$.

(3) $U(x) \pi(a) U(x)^{*}=\pi(T(x) a)$ for all $x$ in $G$ and $a$ in $A$. Such pairs are called representing pairs for $G$ and $A$. The one-to-one correspondence mentioned above is set up by $L(f)=\int_{G} \pi(f(x)) U(x) d x$. In this case $L$ is called the integrated form of $(\pi, U)$. If $L_{1}$ and $L_{2}$ are the integrated forms of $\left(\pi_{1}, U_{1}\right)$ and $\left(\pi_{2}, U_{2}\right)$, respectively, then a mapping $V$ sets up an equivalence between $L_{1}$ and $L_{2}$ if and only if $V$ sets up an equivalence between $\pi_{1}$ and $\pi_{2}$ and between $U_{1}$ and $U_{2}$. For details, see [4].

Let $(T, \alpha)$ be a twisting pair for $G$ and $A$. By [4, Lemma 4.6], $G$ acts on the dual space $\hat{A}$ of $A$ as a topological transformation group by $[\pi]_{x}=$ $\left[\pi_{x}\right]$, where $\pi_{x}(a)=\pi(T(x) a)$ and $[\pi]$ denotes the equivalence class of $\pi$. If $[\pi] \in \hat{A}$, the subgroup $G_{\pi}=\left\{x \in G \mid[\pi]=\left[\pi_{x}\right]\right\}$ is called the stability group of $\pi$ (or of [ $\pi]$ ). By [17, Theorem 2.4], if $A$ is type I, then $G$ acts as a Borel transformation group on $\hat{A}$, and the stability groups are closed subgroups of $G$. If all stability groups are trivial, $G$ is said to act freely on $\hat{A}$.

We will also need the concept of quasi-invariant measures. Let $G$ act on a Borel space $\Gamma$ as a Borel transformation group. Let $\mu$ be a measure on $\Gamma$, and for each $s \in G$, let $s(\mu)$ denote the measure defined by $s(\mu)(E)=\mu(E s)$ for each Borel set $E$ in $\Gamma$, where $E s$ means the elements of $E$ acted upon 
by $s$. If $s(\mu)$ and $\mu$ are equivalent (in the sense of absolute continuity) for all $s \in G, \mu$ is said to be quasi-invariant. If the quasi-invariant measure $\mu$ satisfies $\mu(E)=0$ or $\mu(\Gamma-E)=0$ for every Borel set $E$ of $\Gamma$ with $\mu(E \Delta E s)=0$ for every $s \in G$, then $\mu$ is said to be ergodic, where $\Delta$ here means the symmetric difference of sets. If $\mu$ is concentrated in an orbit, $\mu$ is said to be transitive. We denote by $\lambda$ the Radon-Nikodým derivative of $s(\mu)$ with respect to $\mu$; that is,

$$
\int f(\gamma s) \lambda(\gamma, s) d \mu(\gamma)=\int f(\gamma) d \mu(\gamma)
$$

for all integrable Borel $f$. The function $\lambda$ satisfies the property

$$
\lambda(\gamma, x y)=\lambda(\gamma, x) \lambda(\gamma x, y)
$$

for every $x, y$ in $G$ and almost every $\gamma \in \Gamma$.

3. Induced representations. We give two definitions of induced representation. The first is a general definition that works in all twisted group algebras, and the second makes no sense in the general setting but is extremely useful for our purposes when it does make sense. We show that under special circumstances, the two definitions give rise to equivalent representations, and we obtain other results which are needed in later sections.

Let $(G, A ; T, \alpha)$ be a system such that $A$ is a $C^{*}$-algebra; $G$ is a group; and $(T, \alpha)$ is a twisting pair for $G$ and $A$. Let $(\pi, U)$ be a representing pair for $\left(G_{0}, A ; T, \alpha\right)$ on a Hilbert space $H$, where $G_{0}$ is a closed subgroup of $G$. Let $\mu$ be a quasi-invariant measure on the right coset space $G / G_{0}$, and let $\lambda$ denote the corresponding $\lambda$-function (the Radon-Nikodým derivative of $s(\mu)$ with respect to $\mu$ ). Following the lead of Mackey in [15] and Takesaki in [17], we now construct an induced representing pair for $(G, A ; T, \alpha)$.

Define $\widetilde{H}$ to be all $H$-valued functions on $G$ such that

(a) $(f(x) \mid v)$ is a Borel function of $x$ for every $v \in H$, where $(\cdot \mid \cdot)$ means the inner product in $H$.

(b) $f(x y)=\pi\left(\alpha(x, y)^{*}\right) U(x) f(y)$ for every $x \in G_{0}$ and $y \in G$.

(c) $\int_{G / G_{0}}(f(x) \mid f(x)) d \mu(\dot{x})<\infty$, where $\dot{x}$ is the coset of $x$. The integral in property (c) is well defined because of property (b). Define an inner product on $\widetilde{H}$ by $\left(f_{1} \mid f_{2}\right)=\int\left(f_{1}(x) \mid f_{2}(x)\right) d \mu(\dot{x})$, where we identify functions equal almost everywhere. We then have that $\widetilde{H}$ is a Hilbert space. For each $a \in A$, define an operator $\widetilde{\pi}(a)$ on $\widetilde{H}$ by

$$
(\tilde{\pi}(a) f)(y)=\pi(T(y) a) f(y)
$$

for all $f \in \widetilde{H}, y \in G$. For each $y \in G$, define an operator $\widetilde{U}(y)$ on $\widetilde{H}$ by 


$$
(\widetilde{U}(y) f)(x)=\lambda(x, y)^{1 / 2} \pi(\alpha(x, y)) f(x y)
$$

for all $f \in \widetilde{H}, x \in G$. Note that $\tilde{\pi}(a) f$ and $\widetilde{U}(y) f$ are indeed members of $\widetilde{H}$, for certainly properties (a) and (c) above are satisfied, and if $x \in G_{0}, y \in G$, then

$$
\begin{aligned}
(\tilde{\pi}(a) f)(x y) & =\pi(T(x y) a) f(x y)=\pi(T(x y) a) \pi\left(\alpha(x, y)^{*}\right) U(x) f(y) \\
& =\pi\left([T(x y) a] \alpha(x, y)^{*}\right) U(x) f(y) \\
& =\pi\left(\alpha(x, y)^{*}\right) \pi(T(x) T(y) a) U(x) f(y) \\
& =\pi\left(\alpha(x, y)^{*}\right) U(x) \pi(T(y) a) f(y) \\
& =\pi\left(\alpha(x, y)^{*}\right) U(x)(\tilde{\pi}(a) f)(y) .
\end{aligned}
$$

Also, if $x \in G_{0}, y, z \in G$, then

$$
\begin{aligned}
(\widetilde{U}(z) f)(x y) & =\lambda(x y, z)^{1 / 2} \pi(\alpha(x y, z)) f(x y z) \\
& =\lambda(y, z)^{1 / 2} \pi(\alpha(x y, z)) \pi\left(\alpha(x, y z)^{*}\right) U(x) f(y z) \\
& =\lambda(y, z)^{1 / 2} \pi\left(\alpha(x, y)^{*}[T(x) \alpha(y, z)]\right) U(x) f(y z) \\
& =\lambda(y, z)^{1 / 2} \pi\left(\alpha(x, y)^{*}\right) U(x) \pi(\alpha(y, z)) f(y z) \\
& =\pi\left(\alpha(x, y)^{*}\right) U(x)(\widetilde{U}(z) f)(y),
\end{aligned}
$$

so our assertion follows.

THEOREM 3.1. $(\tilde{\pi}, \widetilde{U})$ is a representing pair for $(G, A ; T, \alpha)$.

Proof. Clearly $\tilde{\pi}$ is a representation of $A$ and $\widetilde{U}(x)$ is unitary for each $x$. Thus we only need to show that (i) $\widetilde{U}(x) \widetilde{U}(y)=\widetilde{\pi}(\alpha(x, y)) \widetilde{U}(x y)$, and (ii) $\widetilde{U}(x) \widetilde{\pi}(a) \widetilde{U}(x)^{*}=\widetilde{\pi}(T(x) a)$. Equality (i) holds, for

$$
\begin{aligned}
(\widetilde{U}(x) \widetilde{U}(y) f)(z) & =\lambda(z, x)^{1 / 2} \pi(\alpha(z, x))(\widetilde{U}(y) f)(z x) \\
& =\lambda(z, x)^{1 / 2} \lambda(z x, y)^{1 / 2} \pi(\alpha(z, x)) \pi(\alpha(z x, y)) f(z x y) \\
& =\lambda(z, x y)^{1 / 2} \pi([T(z) \alpha(x, y)] \alpha(z, x y)) f(z x y) \\
& =\pi(T(z) \alpha(x, y))(\widetilde{U}(x y) f)(z) \\
& =(\pi(\alpha(x, y)) \widetilde{U}(x y) f)(z) .
\end{aligned}
$$

Equality (ii) holds, for 


$$
\begin{aligned}
(\widetilde{U}(x) \tilde{\pi}(a) f)(z) & =\lambda(z, x)^{1 / 2} \pi(\alpha(z, x))(\tilde{\pi}(a) f)(z x) \\
& =\lambda(z, x)^{1 / 2} \pi(\alpha(z, x)) \pi(T(z x) a) f(z x) \\
& =\lambda(z, x)^{1 / 2} \pi(T(z) T(x) a) \pi(\alpha(z, x)) f(z x) \\
& =\pi(T(z) T(x) a)(\widetilde{U}(x) f)(z)=(\tilde{\pi}(T(x) a) \widetilde{U}(x) f)(z) .
\end{aligned}
$$

THEOREM 3.2. If $\left(\pi_{1}, U_{1}\right)$ and $\left(\pi_{2}, U_{2}\right)$ are equivalent representing pairs for $\left(G_{0}, A ; T, \alpha\right)$, then $\left(\tilde{\pi}_{1}, \widetilde{U}_{1}\right)$ and $\left(\widetilde{\pi}_{2}, \widetilde{U}_{2}\right)$ are equivalent.

Proof. Mackey [14, Theorem 2.1] has shown that the choice of quasiinvariant measure is unimportant as far as equivalence is concerned, so we may assume that the same measure is used in obtaining both $\left(\pi_{1}, \widetilde{U}_{1}\right)$ and $\left(\tilde{\pi}_{2}, \widetilde{U}_{2}\right)$. If $V$ sets up an equivalence between $\left(\pi_{1}, U_{1}\right)$ and $\left(\pi_{2}, U_{2}\right)$, then $W$ defined by $(W f)(x)=V(f(x))$ sets up an equivalence between $\left(\widetilde{\pi}_{1}, \widetilde{U}_{1}\right)$ and $\left(\tilde{\pi}_{2}, \widetilde{U}_{2}\right)$, for it is easy to show that $W$ is a unitary between $\widetilde{H}_{1}$ and $\widetilde{H}_{2}$, and

$$
\begin{aligned}
\left(W \widetilde{\pi}_{1}(a) f\right)(x) & =V\left(\left(\tilde{\pi}_{1}(a) f\right)(x)\right)=V\left(\pi_{1}(T(x) a) f(x)\right)=\pi_{2}(T(x) a) V(f(x)) \\
& =\pi_{2}(T(x) a)(W f)(x)=\left(\pi_{2}(a) W f\right)(x) .
\end{aligned}
$$

Also,

$$
\begin{aligned}
\left(W \widetilde{U}_{1}(y) f\right)(x) & =V\left[\left(\widetilde{U}_{1}(y) f\right)(x)\right]=\lambda(x, y)^{1 / 2} V\left[\pi_{1}(\alpha(x, y)) U_{1}(y) f(x y)\right] \\
& =\lambda(x, y)^{1 / 2} \pi_{2}(\alpha(x, y)) U_{2}(y) V(f(x y))=\left(\widetilde{U}_{2}(y) W f\right)(x) .
\end{aligned}
$$

Clearly, if $G_{0}$ is taken to be the trivial subgroup of $G$, then the induced representation is determined by the representation $\pi$ of $A$. In this case, the induced representation is equivalent to the induced representation in the sense of Busby and Smith in [4], and we denote these representations by $I(\pi)$.

We now give a definition of induced representation along the line of Effros and Hahn in [6]. For this, we assume $\alpha$ is continuous. In this case, the space of continuous functions with compact support, equipped with multiplication and involution as in $L^{1}(G, A ; T, \alpha)$, becomes a *-algebra, which we denote by $K(G, A ; T, \alpha)$. Let $\phi$ be a positive linear functional on $K(G, A ; T, \alpha)$. Then $\phi$ defines a pseudo inner product on $K(G, A ; T, \alpha)$ by $(f \mid g)=\phi\left(f^{*} g\right)$. Let $N$ denote the set of functions $f$ such that $(f \mid f)=0$, and let $H_{0}(\phi)$ denote the quotient space $K(G, A ; T, \alpha) / N$. Then $\phi$ defines an inner product on $H_{0}(\phi)$. Denote the completion of $H_{0}(\phi)$ by $H(\phi)$, so that $H(\phi)$ is a Hilbert space. If $g \in K(G, A ; T, \alpha)$, let $g^{\prime}$ denote the equivalence class of $g$ in $H_{0}(\phi)$. For each $f, g \in K(G, A ; T, \alpha)$, define $L(\phi)(f) g^{\prime}=(f g)^{\prime}$. We show that $L(\phi)$ can be extended to a representation of $L^{1}(G, A ; T, \alpha)$ by exhibiting a representing pair $\left(\pi_{\phi}, U_{\phi}\right)$ for which $L(\phi)$ is the integrated form. 
Let $a \in A$. For each $f \in K(G, A ; T, \alpha)$, define $\pi_{\phi}(a)$ by $\pi_{\phi}(a) f^{\prime}=$ $(a f)^{\prime}$ where $(a f)(y)=a f(y)$ for each $y$ in $G$. For each $s$ in $G$, define $U_{\phi}(s)$ by $U_{\phi}(s) f^{\prime}=(s f)^{\prime}$, where $(s f)(y)=\left(T(s) f\left(s^{-1} y\right)\right) \alpha\left(s, s^{-1} y\right)$ for each $y$ in $G$. We will abuse the notation and write $\pi_{\phi}(a) f$ and $U_{\phi}(s) f$ for computational purposes.

LEMMA 3.3. $\left(\pi_{\phi}, U_{\phi}\right)$ as defined above extends uniquely to a representing pair for $(G, A ; T, \alpha)$ on $H(\phi)$.

Proof. We first show that $\pi_{\phi}$ can be extended to a representation of $A$. It is clear that, for each $a$ in $A, \pi_{\phi}(a)$ is a linear operator on $H_{0}(\phi)$. We show that $\pi_{\phi}(a)$ is bounded and hence can be extended to $H(\phi)$. Note that $(a f)^{*}(a f)=f^{*}\left(a^{*} a f\right)$. Let $k=\left(\|a\|^{2}-a^{*} a\right)^{1 / 2}$. (Here we are treating elements of $A$ as though they were in the double centralizer algebra $M(A)$.) The function $k$ exists since $\left\|a^{*} a\right\|=\|a\|^{2}$. Now $k \in M(A)$, so $(k f)$, defined by $(k f)(x)=$ $k f(x)$, is an element of $K(G, A ; T, \alpha)$. For each $x$ in $G$,

$[(k f) *(k f)](x)=\int(k f) *(y)\left(T(y)(k f)\left(y^{-1} x\right)\right) \alpha\left(y, y^{-1} x\right) d y$

$$
\begin{aligned}
= & \int \Delta\left(y^{-1}\right) \alpha\left(y, y^{-1}\right)^{*}\left(T(y)(k f)\left(y^{-1}\right)^{*}\right)\left(T(y)(k f)\left(y^{-1} x\right)\right) \alpha\left(y, y^{-1} x\right) d y \\
= & \int \Delta\left(y^{-1}\right) \alpha\left(y, y^{-1}\right)^{*}\left(T(y)\left(f\left(y^{-1}\right)^{*} k^{*}\right)\right)\left(T(y)\left(k f\left(y^{-1} x\right)\right)\right) \alpha\left(y, y^{-1} x\right) d y \\
= & \int \Delta\left(y^{-1}\right) \alpha\left(y, y^{-1}\right)^{*} T(y)\left(f\left(y^{-1}\right)^{*} k^{*} k f\left(y^{-1} x\right)\right) \alpha\left(y, y^{-1} x\right) d y \\
= & \int \Delta\left(y^{-1}\right) \alpha\left(y, y^{-1}\right)^{*} T(y)\left[f\left(y^{-1}\right)^{*}\left(\|a\|^{2}-a^{*} a\right) f\left(y^{-1} x\right)\right] \alpha\left(y, y^{-1} x\right) d y \\
= & \|a\|^{2} \int \Delta\left(y^{-1}\right) \alpha\left(y, y^{-1}\right)^{*} T(y)\left(f\left(y^{-1}\right)^{*} f\left(y^{-1} x\right)\right) \alpha\left(y, y^{-1} x\right) d y \\
& -\int \Delta\left(y^{-1}\right) \alpha\left(y, y^{-1}\right)^{*}\left(T(y) f\left(y^{-1}\right)^{*}\right) T(y)\left(a^{*} a\left(f\left(y^{-1} x\right)\right)\right) \alpha\left(y, y^{-1} x\right) d y \\
= & \|a\|^{2} f^{*} f(x)-f^{*}\left(a^{*} a f\right)(x) .
\end{aligned}
$$

Hence $(a f)^{*}(a f)+(k f)^{*}(k f)=\|a\|^{2} f^{*} f$, so $\left\|(a f)^{\prime}\right\|_{\phi}^{2}+\left\|(k f)^{\prime}\right\|_{\phi}^{2}=\|a\|^{2}\left\|f^{\prime}\right\|_{\phi}^{2}$. Then $\left\|(a f)^{\prime}\right\|_{\phi} \leqslant\|a\|\left\|f^{\prime}\right\|_{\phi}$ and $\pi_{\phi}(a)$ is continuous. Now for $g, f$ in $K(G, A ; T, \alpha), g^{*}(a f)=\left(a^{*} g\right) * f$, so

$$
\left(\pi_{\phi}(a) f^{\prime} \mid g\right)=\phi\left(g^{*}(a f)\right)=\phi\left(\left(a^{*} g\right)^{*} f\right)=\left(f^{\prime} \mid\left(a^{*} g\right)^{\prime}\right)_{\phi}=\left(f^{\prime} \mid \pi_{\phi}\left(a^{*}\right) g^{\prime}\right)_{\phi} .
$$

Thus $\pi_{\phi}$ becomes a representation of $A$ on $H(\phi)$.

We now show that for each $s$ in $G, U_{\phi}(s)$ is unitary. First note that for $h \in K(G, A ; T, \alpha)$ and $y$ in $G$,

$$
\begin{aligned}
\left(U_{\phi}(s) h\right)^{*}(y) & =\alpha\left(y, y^{-1}\right)^{*}\left[T(y)\left(\left(U_{\phi}(s) h\right)\left(y^{-1}\right)^{*}\right)\right] \Delta\left(y^{-1}\right) \\
& =\alpha\left(y, y^{-1}\right)^{*} T(y)\left[T(s) h\left(s^{-1} y^{-1}\right) \alpha\left(s, s^{-1} y^{-1}\right)\right]^{*} \Delta\left(y^{-1}\right) \\
& =\alpha\left(y, y^{-1}\right) * T(y)\left(\alpha\left(s, s^{-1} y^{-1}\right)^{*} T(s) h\left(s^{-1} y^{-1}\right)^{*}\right) \Delta\left(y^{-1}\right) .
\end{aligned}
$$

Thus, for each $h, g$ in $K(G, A ; T, \alpha)$, 
$\left(U_{\phi}(s) h\right) *\left(U_{\phi}(s) g\right)(x)$

$$
\begin{gathered}
=\int\left\{\alpha\left(y, y^{-1}\right)^{*} T(y)\left(\alpha\left(s, s^{-1} y^{-1}\right)^{*} T(s) h\left(s^{-1} y^{-1}\right)^{*}\right) \Delta\left(y^{-1}\right)\right\} \\
\cdot\left\{T(y)\left[T(s) g\left(s^{-1} y^{-1} x\right) \alpha\left(s, s^{-1} y^{-1} x\right)\right] \alpha\left(y, y^{-1} x\right)\right\} d y \\
=\int \alpha\left(y, y^{-1}\right)^{*} T(y) \alpha\left(s, s^{-1} y^{-1}\right)^{*} \alpha(y, x) T(y x) h\left((y s)^{-1}\right)^{*} \\
\quad \cdot T(y s) g\left((y s)^{-1} x\right) \alpha(y, s)^{*} T(y) \alpha\left(s,(y s)^{-1} x\right) \alpha\left(y, y^{-1} x\right) d y \\
=\int \Delta(s) \alpha\left(y, y^{-1}\right)^{*} T(y) \alpha\left(s,(y s)^{-1}\right)^{*} \alpha(y, s) \alpha\left(y s, s^{-1} y^{-1}\right) h^{*}(y s) \\
\quad \cdot T(y s) g\left((y s)^{-1} x\right) \alpha(y, x)^{*} T(y) \alpha\left(s,(y s)^{-1} x\right) \alpha\left(y, y^{-1} x\right) d y \\
=\int \alpha\left(y s^{-1}, s y^{-1}\right)^{*} T\left(y s^{-1}\right) \alpha\left(s, y^{-1}\right)^{*} \alpha\left(y s^{-1}, s\right) \alpha\left(y, y^{-1}\right) h^{*}(y) \\
\quad \cdot T(y) g\left(y^{-1} x\right) \alpha\left(y s^{-1}, s\right)^{*} T\left(y s^{-1}\right) \alpha\left(s, y^{-1}\right) \alpha\left(y s^{-1}, s y^{-1} x\right) d y \\
=\int h^{*}(y)\left(T(y) g\left(y^{-1} x\right)\right) \alpha\left(y s^{-1}, s\right)^{*} T\left(y s^{-1}\right) \alpha\left(s, y^{-1} x\right) \alpha\left(y s^{-1}, s y^{-1} x\right) d y \\
=\int h^{*}(y)\left(T(y) g\left(y^{-1} x\right)\right) \alpha\left(y, y^{-1} x\right) d y=h^{*} g(x) .
\end{gathered}
$$

Hence $\left(U_{\phi}(s) g^{\prime} \mid U_{\phi}(s) h^{\prime}\right)_{\phi}=\left(g^{\prime} \mid h^{\prime}\right)_{\phi}$ and $U_{\phi}(s)$ is unitary and extends to a unitary operator on $H(\phi)$.

Finally, it is easy to show that $U_{\phi}(x) U_{\phi}(y)=\pi_{\phi}(\alpha(x, y)) U_{\phi}(x y)$ and $U_{\phi}(x) \pi_{\phi}(a) U_{\phi}(x)^{*}=\pi_{\phi}(T(x) a)$ for every $x, y$ in $G$ and $a$ in $A$. Therefore $\left(\pi_{\phi}, U_{\phi}\right)$ is indeed a representing pair.

LEMMA 3.4. With notation as before, $L(\phi)$ is the integrated form of $\left(\pi_{\phi}, U_{\phi}\right)$ and hence extends uniquely to a representation of $L^{1}(G, A ; T, \alpha)$ on $H(\phi)$.

Proof. For each $f, g$ in $K(G, A ; T, \alpha)$ and $x$ in $G$,

$$
\begin{aligned}
(f g)(x) & =\int f(y)\left(T(y) g\left(y^{-1} x\right)\right) \alpha\left(y, y^{-1} x\right) d y \\
& =\int\left(\pi_{\phi}(f(y)) U_{\phi}(y) g\right)(x) d y,
\end{aligned}
$$

so $L(\phi)(f)=\int \pi_{\phi}(f(y)) U_{\phi}(y) d y$.

We now define $\phi$ in a special way and show that the resulting $L(\phi)$ is unitarily equivalent to the induced representation in our sense. Let $\pi$ be a representation of $A$ on $H$, and let $v \in H$ with $\|v\|=1$. For each $f$ in $K(G, A ; T, \alpha)$, define $\phi(f)=(\pi(f(e)) v \mid v)$. The function $\phi$ is clearly a linear functional on $K(G, A ; T, \alpha)$. We have

$$
\begin{aligned}
f^{*} f(e) & =\int f^{*}(y)\left(T(y) f\left(y^{-1}\right)\right) \alpha\left(y, y^{-1}\right) d y \\
& =\int \Delta\left(y^{-1}\right)\left[\left(T(y) f\left(y^{-1}\right)\right) \alpha\left(y, y^{-1}\right)\right]^{*}\left[\left(T(y) f\left(y^{-1}\right)\right) \alpha\left(y, y^{-1}\right)\right] d y .
\end{aligned}
$$


Thus,

$\phi\left(f^{*} f\right)=\int \Delta\left(y^{-1}\right)\left(\pi\left[\left(T(y) f\left(y^{-1}\right)\right) \alpha\left(y, y^{-1}\right)\right] v \mid \pi\left[\left(T(y) f\left(y^{-1}\right)\right) \alpha\left(y, y^{-1}\right)\right] v\right) d y \geqslant 0$.

We may therefore use $\phi$ to define a representation $L(\phi)$ of $L^{1}(G, A ; T, \alpha)$ on $H(\phi)$.

THEOREM 3.5. Let $\pi$ be an irreducible representation of $A$ on a Hilbert space $H$. Let $v \in H$ with $\|v\|=1$. Define $\phi(f)=(\pi(f(e)) v \mid v)$ for each $f \in$ $K(G, A ; T, \alpha)$. Let $L(\phi)$ denote the representation of $L^{1}(G, A ; T, \alpha)$ defined by $\phi$. If the stability group of $\pi$ is trivial, then $I(\pi)$ and $L(\phi)$ are unitarily equivalent.

ProOF. The Hilbert space of $I(\pi)$ is $L^{2}(G, H, \mu)$. Define $W: K(G, A)$ $\rightarrow L^{2}(G, H, \mu)$ by

$$
W(f)(x)=\lambda\left(x, x^{-1}\right)^{1 / 2} \pi\left(\left(T(x) f\left(x^{-1}\right)\right) \alpha\left(x, x^{-1}\right)\right) v .
$$

We show that the closure of $W$ sets up the desired equivalence. Note that

$$
\lambda\left(s t, t^{-1} s^{-1}\right) \lambda(s, t)=\frac{\lambda\left(s, s^{-1}\right)}{\lambda(s, t)} \lambda(s, t)=\lambda\left(s, s^{-1}\right)
$$

Now

$$
\int f(s t) \lambda\left(s t, t^{-1} s^{-1}\right) \lambda(s, t) d \mu(s)=\int f(s) \lambda\left(s, s^{-1}\right) d \mu(s) .
$$

But

$$
\int f(s t) \lambda\left(s t, t^{-1} s^{-1}\right) \lambda(s, t) d \mu(s)=\int f(s t) \lambda\left(s, s^{-1}\right) d \mu(s) .
$$

But this means that $\lambda\left(s, s^{-1}\right) d \mu(s)$ is a right-invariant measure, so $\lambda\left(s, s^{-1}\right) d \mu(s)$ $=\Delta\left(s^{-1}\right) d s$. (See $[16$, p. 36].) Let $f, g$ be in $K(G, A ; T, \alpha)$. Then

$$
\begin{aligned}
(W(f) \mid W(g))=\int(W(f)(x) \mid W(g)(x)) d \mu(x) \\
=\int\left(\lambda\left(x, x^{-1}\right)^{1 / 2} \pi\left(T(x) f\left(x^{-1}\right) \alpha\left(x, x^{-1}\right)\right) v \mid \lambda\left(x, x^{-1}\right)^{1 / 2}\right. \\
\left.\cdot \pi\left(T(x) g\left(x^{-1}\right) \alpha\left(x, x^{-1}\right)\right) v\right) d \mu(x) \\
=\int\left(\pi\left[\lambda\left(x, x^{-1}\right)\left(T(x) g\left(x^{-1}\right) \alpha\left(x, x^{-1}\right)\right)^{*}\left(T(x) f\left(x^{-1}\right) \alpha\left(x, x^{-1}\right)\right)\right] v \mid v\right) d \mu(x) \\
=\left(\pi\left[\int \lambda\left(x, x^{-1}\right) \alpha\left(x, x^{-1}\right)^{*}\left(T(x) g\left(x^{-1}\right)^{*}\right)\left(T(x) f\left(x^{-1}\right)\right) \alpha\left(x, x^{-1}\right) d \mu(x)\right] v \mid v\right) \\
=\left(\pi\left[\int \Delta\left(x^{-1}\right) \alpha\left(x, x^{-1}\right)^{*}\left(T(x) g\left(x^{-1}\right)^{*}\right)\left(T(x) f\left(x^{-1}\right)\right) \alpha\left(x, x^{-1}\right) d x\right] v \mid v\right) \\
=\left(\pi\left[\int g^{*}(x)\left(T(x) f\left(x^{-1}\right)\right) \alpha\left(x, x^{-1}\right) d x\right] v \mid v\right) \\
=\left(\pi\left(g^{*} f(e)\right) v \mid v\right)=\phi\left(g^{*} f\right)=(f \mid g)_{\phi} .
\end{aligned}
$$


Hence $W$ is unitary if $\widetilde{U}(s) W=W U_{\phi}(s)$ and $\tilde{\pi}(a) W=W \pi_{\phi}(a)$, where $L(\phi)$ and $I(\pi)$ are the integrated forms of $\left(\pi_{\phi}, U_{\phi}\right)$ and $(\tilde{\pi}, \widetilde{U})$, respectively. This is because $I(\pi)$ is irreducible. But

$$
\begin{aligned}
(\tilde{U}(s) W f)(y) & =\lambda(y, s)^{1 / 2} \pi(\alpha(y, s))(W f)(y s) \\
& =\lambda(y, s)^{1 / 2} \lambda\left(y s, s^{-1} y^{-1}\right)^{1 / 2} \pi\left(\alpha(y, s)\left(T(y s) f\left(s^{-1} y^{-1}\right)\right) \alpha\left(y s, s^{-1} y^{-1}\right)\right) v \\
& =\lambda\left(y, y^{-1}\right)^{1 / 2} \pi\left(\left(T(y) T(s) f\left(s^{-1} y^{-1}\right)\right) \alpha(y, s) \alpha\left(y s, s^{-1} y^{-1}\right)\right) v \\
& =\lambda\left(y, y^{-1}\right)^{1 / 2} \pi\left(T(y)\left[T(s) f\left(s^{-1} y^{-1}\right) \alpha\left(s, s^{-1} y^{-1}\right)\right] \alpha\left(y, y^{-1}\right)\right) v \\
& =\lambda\left(y, y^{-1}\right)^{1 / 2} \pi\left(T(y)(U(s) f)\left(y^{-1}\right) \alpha\left(y, y^{-1}\right)\right) v \\
& =\left(W U_{\phi}(s) f\right)(y)
\end{aligned}
$$

and

$$
\begin{aligned}
& (\tilde{\pi}(a) W f)(y)=\pi(T(y) a) \lambda\left(y, y^{-1}\right)^{1 / 2} \pi\left(T(y) f\left(y^{-1}\right) \alpha\left(y, y^{-1}\right)\right) v \\
& =\lambda\left(y, y^{-1}\right)^{1 / 2} \pi\left(T(y)(a f)\left(y^{-1}\right) \alpha\left(y, y^{-1}\right)\right) v \\
& =\lambda\left(y, y^{-1}\right)^{1 / 2} \pi\left(T(y)\left(\pi_{\phi}(a) f\right)\left(y^{-1}\right) \alpha\left(y, y^{-1}\right)\right) v=\left(W \pi_{\phi}(a) f\right)(y) .
\end{aligned}
$$

Hence $W$ sets up the desired equivalence.

4. Orbits of representations. We now investigate the relationship between induced representations and orbits in the dual space $\hat{A}$ of $A$. If $\pi$ is an irreducible representation of $A$, then the orbit of $[\pi]$ in $\hat{A}$ is $\{[\pi \circ T(x)] \mid$ $x \in G\}$ where here the square brackets indicate equivalence class.

Lemma 4.1. Let $G_{0}$ be a closed subgroup of $G$ and let $(\pi, U)$ be a representing pair for $\left(G_{0}, A ; T, \alpha\right)$. Then for each $s \in G$, the pair $\left(\pi_{s}, U_{s}\right)$ defined by $\pi_{s}(a)=\pi(T(s) a)$ and $U_{s}(x)=\pi\left(\alpha(s, x) \alpha\left(s x s^{-1}, s\right)^{*}\right) U\left(s x s^{-1}\right)$ is a representing pair for $\left(s^{-1} G_{0} s, A ; T, \alpha\right)$.

Proof. Let $s \in G$ and $x=s^{-1} x_{0} s$, where $x_{0} \in G_{0}$. Then

$$
\begin{aligned}
U_{s}(x) \pi(T(s) a) U_{s}(x)^{*}=\pi\left(\alpha(s, x) \alpha\left(x_{0}, s\right)^{*}\right) U\left(x_{0}\right) \pi(T(s) a) U\left(x_{0}\right)^{*} \pi\left(\alpha\left(x_{0}, s\right) \alpha(s, x)^{*}\right) \\
\quad=\pi(\alpha(s, x)) \pi\left(\alpha\left(x_{0}, s\right)^{*}\left[T\left(x_{0}\right) T(s) a\right] \alpha\left(x_{0}, s\right)\right) \pi\left(\alpha(s, x)^{*}\right) \\
=\pi\left(\alpha(s, x)\left[T\left(x_{0}, s\right) a\right] \alpha(s, x)^{*}\right)=\pi\left(\alpha(s, x)[T(s x) a] \alpha(s, x)^{*}\right) \\
=\pi(T(s) T(x) a)=\pi_{s}(T(x) a) .
\end{aligned}
$$

If $y=x^{-1} y_{0} s, y_{0} \in G_{0}$, then 


$$
\begin{aligned}
& \pi_{s}(\alpha(x, y)) U_{s}(x y)=\pi(T(s) \alpha(x, y)) \pi\left(\alpha(s, x y) \alpha\left(x_{0} y_{0}, s\right)^{*}\right) U\left(x_{0} y_{0}\right) \\
& \quad=\pi\left(\alpha(s, x) \alpha(s x, y) \alpha\left(x_{0} y_{0}, s\right)^{*} \alpha\left(x_{0}, y_{0}\right)^{*}\right) U\left(x_{0}\right) U\left(y_{0}\right) \\
& \quad=\pi\left(\alpha(s, x) \alpha(s x, y)\left\{\left[T\left(x_{0}\right) \alpha\left(y_{0}, s\right)\right] \alpha\left(x_{0}, y_{0} s\right)\right\}^{*}\right) U\left(x_{0}\right) U\left(y_{0}\right) \\
& \quad=\pi\left(\alpha(s, x) \alpha(s x, y) \alpha\left(x_{0}, y_{0} s\right)^{*} T\left(x_{0}\right) \alpha\left(y_{0}, s\right)^{*}\right) U\left(x_{0}\right) U\left(y_{0}\right) \\
& =\pi\left(\alpha(s, x) \alpha(s x, y) \alpha\left(x_{0}, y_{0} s\right)^{*}\right) U\left(x_{0}\right) \pi\left(\alpha\left(y_{0}, s\right)^{*}\right) U\left(y_{0}\right) \\
& \quad=\pi\left(\alpha(s, x) \alpha(s x, y) \alpha\left(x_{0}, y_{0} s\right)^{*}\right) U\left(x_{0}\right) \pi\left(\alpha(s, y)^{*}\right) \pi\left(\alpha(s, y) \alpha\left(y_{0}, s\right)^{*}\right) U\left(y_{0}\right) \\
& \quad=\pi\left(\alpha(s, x) \alpha(s x, y) \alpha\left(x_{0}, y_{0} s\right)^{*}\left[T\left(x_{0}\right) \alpha(s, y)^{*}\right]\right) U\left(x_{0}\right) U_{s}(y) .
\end{aligned}
$$

But

$$
\begin{aligned}
& \alpha(s x, y) \alpha\left(x_{0}, y_{0} s\right)^{*}\left[T\left(x_{0}\right) \alpha(s, y)^{*}\right] \\
& \quad=\alpha\left(x_{0}, s\right)^{*}\left[T\left(x_{0}\right) \alpha(s, y)\right] \alpha\left(x_{0}, y_{0} s\right) \alpha\left(x_{0}, y_{0} s\right)^{*}\left[T\left(x_{0}\right) \alpha(s, y)^{*}\right] \\
& \quad=\alpha\left(x_{0}, s\right) *
\end{aligned}
$$

Thus, $\pi_{s}(\alpha(x, y)) U_{s}(x y)=U_{s}(x) U_{s}(y)$, so $\left(\pi_{s}, U_{s}\right)$ is a representing pair.

LEMMA 4.2. Let $G_{0},(\pi, U)$ and $\left(\pi_{s}, U_{s}\right)$ be as in the previous lemma. Then $(\tilde{\pi}, \widetilde{U})$ is unitarily equivalent to $\left(\tilde{\pi}_{s}, \widetilde{U}_{s}\right)$.

Proof. We shall denote $s^{-1} G_{0} s$ by $G_{0}(s)$. Let $\mu$ be the quasi-invariant measure used to obtain $(\tilde{\pi}, \widetilde{U})$ and let $\lambda$ be its associated $\lambda$-function. Let $\phi$ be the 1-1 Borel set preserving mapping from $G / G_{0}(s)$ onto $G / G_{0}$ defined by $\phi(\dot{t})=G_{0}$ st. For each Borel set $E$ in $G / G_{0}(s)$, define $\mu^{\prime}(E)=\mu(\phi(E))$. Now $\phi(E x)=\phi(E) x$ for every $x \in G$, so $\mu^{\prime}$ is a quasi-invariant measure on $G / G_{0}(s)$, and its associated $\lambda$-function is defined by $\lambda^{\prime}(\dot{t}, x)=\lambda(\phi(\dot{t}), x)$; i.e., $\lambda^{\prime}(\dot{t}, x)=$ $\lambda(s i, x)$. Thus we may use $\mu^{\prime}$ to obtain $\left(\tilde{\pi}_{s}, \widetilde{U}_{s}\right)$.

Let $\widetilde{H}$ and $\widetilde{H}_{s}$ denote the Hilbert spaces of the induced representing pairs $(\tilde{\pi}, \widetilde{U})$ and $\left(\tilde{\pi}_{s}, \widetilde{U}_{s}\right)$, respectively. For each $f \in \widetilde{H}_{s}$, define $h_{f}(t)=$ $\pi\left(\alpha\left(s, s^{-1} t\right)^{*}\right) f\left(s^{-1} t\right)$. We now show that $h_{f} \in \tilde{H}$. Let $x \in G_{0}, y \in G$. Then

$$
\begin{aligned}
h_{f}(x)= & \pi\left(\alpha\left(s, s^{-1} x y\right)^{*}\right) f\left(s^{-1} x y\right)=\pi\left(\alpha\left(s, s^{-1} x y\right)^{*}\right) f\left(s^{-1} x s s^{-1} y\right) \\
= & \pi\left(\alpha\left(s, s^{-1} x y\right)^{*}\right) \pi\left(T(s) \alpha\left(s^{-1} x s, s^{-1} y\right)^{*}\right) U_{s}\left(s^{-1} x s\right) f\left(s^{-1} y\right) \\
= & \pi\left(\alpha\left(s, s^{-1} x y\right)^{*}\right) \pi\left(T(s) \alpha\left(s^{-1} x s, s^{-1} y\right)^{*}\right) \pi\left(\alpha\left(s, s^{-1} x s\right) \alpha(x, s)^{*}\right) U(x) f\left(s^{-1} y\right) \\
= & \pi\left(\alpha\left(s, s^{-1} x y\right)^{*}\left[T(s) \alpha\left(s^{-1} x s, s^{-1} y\right)^{*}\right] \alpha\left(s, s^{-1} x s\right) \alpha(x, s)^{*}\right) U(x) \\
& \cdot \pi\left(\alpha\left(s, s^{-1} y\right)\right) h_{f}(y)
\end{aligned}
$$




$$
\begin{aligned}
= & \pi\left(\alpha\left(s, s^{-1} x y\right)^{*}\left[T(s) \alpha\left(s^{-1} x s, s^{-1} y\right)^{*}\right]\right. \\
& \left.\cdot \alpha\left(s, s^{-1} x s\right) \alpha(x, s)^{*}\left[T(x) \alpha\left(s, s^{-1} y\right)\right]\right) U(x) h_{f}(y) \\
= & \pi\left(\alpha\left(x s, s^{-1} y\right)^{*} \alpha(x, s)^{*}\left[T(x) \alpha\left(s, s^{-1} y\right)\right]\right) U(x) h_{f}(y) \\
= & \pi\left(\left[T(x) \alpha\left(s, s^{-1} y\right)\right] \alpha(x, y)\right)^{*} \pi\left(T(x) \alpha\left(s, s^{-1} y\right)\right) U(x) h_{f}(y) \\
= & \pi\left(\alpha(x, y)^{*}\right) U(x) h_{f}(y) .
\end{aligned}
$$

Also

$$
\begin{aligned}
\int_{G / G_{0}}\left(h_{f}(x) \mid h_{f}(x)\right) d \mu(\dot{x}) & =\int_{G / G_{0}}\left(f\left(s^{-1} x\right) \mid f\left(s^{-1} x\right)\right) d \mu(\dot{x}) \\
& =\int_{G / G_{0}(s)}\left(f\left(s^{-1} x\right) \mid f\left(s^{-1} x\right)\right) d \mu^{\prime}\left(s^{-1} \dot{x}\right) \\
& =\int_{G / G_{0}(s)}(f(t) \mid f(t)) d \mu^{\prime}(\dot{t}) .
\end{aligned}
$$

Thus, $h_{f} \in \widetilde{H}$.

Now, for each $f \in \widetilde{H}_{s}$, define $V: \widetilde{H}_{s} \rightarrow \widetilde{H}$ by $V f=h_{f}$. We show that $V$ is the desired unitary mapping. $V$ is onto, for if $h \in \widetilde{H}$, then $V f=h$ where $f$ is defined by $f(x)=\pi(\alpha(s, x)) h(s x)$. We thus have that $V$ is a unitary mapping from $\widetilde{H}_{s}$ onto $\widetilde{H}$. Let $f \in \widetilde{H}_{s}$.

$$
\begin{aligned}
(\tilde{\pi}(a) V f)(t) & =\pi(T(t) a) \pi\left(\alpha\left(s, s^{-1} t\right)^{*}\right) f\left(s^{-1} t\right) \\
& =\pi\left(\alpha\left(s, s^{-1} t\right)^{*}\right) \pi\left(T(s) T\left(s^{-1} t\right) a\right) f\left(s^{-1} t\right) \\
& =\pi\left(\alpha\left(s, s^{-1} t\right)^{*}\right) \pi_{s}\left(T\left(s^{-1} t\right) a\right) f\left(s^{-1} t\right) \\
& =\pi\left(\alpha\left(s, s^{-1} t\right)^{*}\right)\left(\tilde{\pi}_{s}(a) f\right)\left(s^{-1} t\right) \\
& =\left(V \tilde{\pi}_{s}(a) f\right)(t) .
\end{aligned}
$$

Also

$$
\begin{aligned}
(\widetilde{U}(x) V f)(t) & =\lambda(t, x)^{1 / 2} \pi(\alpha(t, x))(V f)(t x) \\
& =\lambda(t, x)^{1 / 2} \pi(\alpha(t, x)) \pi\left(\alpha\left(s, s^{-1} t x\right)^{*}\right) f\left(s^{-1} t x\right)
\end{aligned}
$$

and

$$
\begin{aligned}
\left(V \widetilde{U}_{s}(x) f\right)(t) & =\pi\left(\alpha\left(s, s^{-1} t\right)^{*}\right)\left(\widetilde{U}_{s}(x) f\right)\left(s^{-1} t\right) \\
& =\lambda^{\prime}\left(s^{-1} t, x\right)^{1 / 2} \pi\left(\alpha\left(s, s^{-1} t\right)^{*}\right) \pi\left(T(s) \alpha\left(s^{-1} t, x\right)\right) f\left(s^{-1} t x\right) \\
& =\lambda(t, x)^{1 / 2} \pi\left(\alpha\left(s, s^{-1} t\right)^{*} \alpha\left(s, s^{-1} t\right) \alpha(t, x) \alpha\left(s, s^{-1} t x\right)^{*}\right) f\left(s^{-1} t x\right) \\
& =\lambda(t, x)^{1 / 2} \pi\left(\alpha(t, x) \alpha\left(s, s^{-1} t x\right)^{*}\right) f\left(s^{-1} t x\right) .
\end{aligned}
$$

This completes the proof. 
COROLLARY 4.3. The induced representation $I\left(\pi_{s}\right)$ is unitarily equivalent to $I(\pi)$ for every $s \in G$. In particular, if $\pi$ is irreducible, then every element in the orbit of $[\pi]$ induces the same (up to equivalence) representation.

We now turn our attention to the relationship of $I(\pi)$ to $I\left(\pi_{0}\right)$, where $\pi_{0}$ is an element in the support of $\pi$. We show that, under special circumstances, $I(\pi)$ and $I\left(\pi_{0}\right)$, as well as another related representation, are weakly equivalent. Suppose $A$ is type $\mathrm{I}$ and $(\pi, U)$ is a factor representing pair for $(G, A ; T, \alpha)$ on $H_{\pi}$. Then, as shown in [17], there exist a Hilbert space $H$ and an ergodic, quasi-invariant measure $\mu$ on $\hat{A}$ such that $H_{\pi}=L^{2}(\hat{A}, H, \mu)$ and $\pi=$ $\int_{\hat{A}}^{\oplus} \pi_{\gamma} d \mu(\gamma)$ where, for almost every $\gamma, \pi_{\gamma}$ is a factor representation which is quasi-equivalent to an element of $\gamma$. Furthermore, by [5, §8.6.9], the support of $\pi$ is the support of $\mu$. With this in mind, we state the following lemmas.

Lemma 4.4. If $A$ is type I and $\mu$ is an ergodic, quasi-invariant measure on the dual space $\hat{A}$ of $A$, then the support of $\mu$ is an orbit closure in $\hat{A}$.

Proof. The proof of [6, Lemma 1.1] and the discussion preceding [6, Theorem 1.3] hold in the more general setting, so the lemma is true.

For every representation $L$, let ker $L$ denote the kernel of $L$. Recall that $C^{*}(G, A ; T, \alpha)$ denotes the enveloping $C^{*}$-algebra of $L^{1}(G, A ; T, \alpha)$.

Lemma 4.5. Suppose $(T, \alpha)$ is a smooth representing pair, and let $\pi$ be a representation of $A$ such that the support of $\pi$ is an orbit closure, say $\overline{\left[\pi_{0}\right] G}$. Then $I(\pi)$ and $I\left(\pi_{0}\right)$ are weakly equivalent.

Proof. Since $\pi_{0} \in$ support of $\pi, \pi_{0}$ is weakly contained in $\pi$. Thus, by $[3$, Corollary 1$], I\left(\pi_{0}\right)$ is weakly contained in $I(\pi)$.

Now $\pi$ is weakly contained in its support, so $I(\pi)$ is weakly contained in $I \overline{\left(\left[\pi_{0}\right] G\right)}$. Let $\zeta_{1} \in \overline{\left[\pi_{0}\right] G}$. Then, by [5, Theorem 3.4.10], $\zeta_{1}$ is weakly contained in $\left[\pi_{0}\right] G$. Thus $I\left(\zeta_{1}\right)$ is weakly contained in $I\left(\left[\pi_{0}\right] G\right)$. But by Lemmas 4.1 and $4.2, I\left(\left[\pi_{0}\right] G\right)$, up to equivalence, is just $I\left(\pi_{0}\right)$. Hence $I\left(\zeta_{1}\right)$ is weakly contained in $I\left(\pi_{0}\right)$. Let $I(\zeta)$ also denote the corresponding representation of $C^{*}(G, A ; T, \alpha)$. Then we have

(1) $I(\pi)$ is weakly contained in $\overline{I\left(\left[\pi_{0}\right] G\right)}$ on $C^{*}(G, A ; T, \alpha)$, and

(2) if $\zeta_{1} \in \overline{\left[\pi_{0}\right] G}, I\left(\zeta_{1}\right)$ is weakly contained in $I\left(\pi_{0}\right)$ on $C^{*}(G, A ; T, \alpha)$. The above two statements (since we are now working on $C^{*}(G, A ; T, \alpha)$ ) are equivalent to saying

$$
\operatorname{ker} I(\pi) \supseteq \frac{\cap}{\left[\pi_{0}\right] G} \operatorname{ker} I(\zeta) \text {, and }
$$

$$
\operatorname{ker} I\left(\zeta_{1}\right) \supseteq \operatorname{ker} I\left(\pi_{0}\right) \text { if } \zeta_{1} \in \overline{\left[\pi_{0}\right] G} \text {. }
$$


Now $\pi_{0} \in \overline{\left[\pi_{0}\right] G}$, so $\overline{\left[\pi_{0}\right] G} \operatorname{ker} I(\zeta)=\operatorname{ker} I\left(\pi_{0}\right)$ because of (2). Thus by (1), $\operatorname{ker} I(\pi) \supseteq \operatorname{ker} I\left(\pi_{0}\right)$ on $C^{*}(G, A ; T, \alpha)$; that is, $I(\pi)$ is weakly contained in $I\left(\pi_{0}\right)$.

We immediately have the following corollary.

COROLLARY 4.6. With notations as in Lemma 4.5, $\operatorname{ker} I(\pi)=\operatorname{ker} I\left(\pi_{0}\right)$.

Let $(\pi, U)$ be a representing pair for $(G, A ; T, \alpha)$ on the Hilbert space $H$. The Hilbert space of $I(\pi)$ is $L^{2}(G, H, \mu)$, where $\mu$ is a finite measure on $G$ with the same null sets as Haar measure. Let $U_{L}$ denote the representation of $G$ induced from the one-dimensional identity representation of $\{e\}$ using the measure $\mu$. (Thus $U_{L}$ is the left regular.representation of $G$.) Let $L^{\otimes}$ denote the integrated form of $\left(\pi \otimes I, U \otimes U_{L}\right)$ where $I$ denotes the identity operator.

THEOREM 4.7. With notation as above, $I(\pi)$ is unitarily equivalent to $L^{\otimes}$.

Proof. The Hilbert space of $L^{\otimes}$ is $H \otimes L^{2}(G, \mu)$. Define $W$ : $H \otimes L^{2}(G, \mu) \rightarrow L^{2}(G, H, \mu)$ by $W(f)(z)=U(z) V(f)(z)$ for every $z$ in $G$, where $V$ is the unitary mapping of $H \otimes L^{2}(G, \mu)$ onto $L^{2}(G, H, \mu)$ which satisfies $V(h \otimes g)(z)=g(z) h$ for every $h \in H$ and $g \in L^{2}(G, \mu)$. (See [5, p. 153, Corollary].) We show $W$ sets up the desired equivalence. $W$ is a unitary mapping, for $W$ is clearly linear and for every $f_{1}, f_{2} \in H \otimes L^{2}(G, \mu)$,

$$
\begin{aligned}
\left(W\left(f_{1}\right) \mid W\left(f_{2}\right)\right) & =\int\left(W\left(f_{1}\right)(z) \mid W\left(f_{2}\right)(z)\right) d z=\int\left(U(z) V\left(f_{1}\right)(z) \mid U(z) V\left(f_{2}(z)\right) d z\right. \\
& =\int\left(V\left(f_{1}\right)(z) \mid V\left(f_{2}\right)(z)\right) d z=\left(V\left(f_{1}\right) \mid V\left(f_{2}\right)\right)=\left(f_{1} \mid f_{2}\right) .
\end{aligned}
$$

Also, for every $a \in A, h \in H, g \in L^{2}(G, \mu)$ and $z \in G$,

$$
\begin{gathered}
W(\pi(a) \otimes I)(h \otimes g)(z)=W(\pi(a) h \otimes g)(z)=g(z) U(z) \pi(a) h=g(z) \pi(T(z) a) U(z) h \\
=\pi(T(z) a)(g(z) U(z) h)=\pi(T(z) a)(W(h \otimes g)(z))=(\tilde{\pi}(a) W(h \otimes g))(z)
\end{gathered}
$$

and for $s$ in $G$,

$$
\begin{aligned}
W\left(U(s) \otimes U_{L}(s)\right)(h \otimes g)(z) & =W\left(U(s) h \otimes U_{L}(s) g\right)(z)=\left(U_{L}(s) g\right)(z) U(z) U(s) h \\
& =\lambda(z, s)^{1 / 2} g(z s) U(z) U(s) h \\
& =\lambda(z, s)^{1 / 2} g(z s) \pi(\alpha(z, s)) U(z s) h \\
& =\lambda(z, s)^{1 / 2} \pi(\alpha(z, s)) g(z s) U(z s) h \\
& =\lambda(z, s)^{1 / 2} \pi(\alpha(z, s)) W(h \otimes g)(z s) \\
& =(\widetilde{U}(s) W(h \otimes g))(z) .
\end{aligned}
$$


Since elements of the form $h \otimes g$ are dense in $H \otimes L^{2}(G, \mu), W$ sets up the desired equivalence.

COROLlaRY 4.8. With notation as in Lemma 4.5 and Theorem 4.7, $I\left(\pi_{0}\right)$ is weakly equivalent to $L^{\otimes}$. (Hence $\operatorname{ker} I\left(\pi_{0}\right)=\operatorname{ker} L^{\otimes}$.)

In [15], G. W. Mackey showed that under special circumstances (i.e., when the orbit space of $G$ is countably separated) all representations of $G$ (up to equivalence) may be obtained by inducing from certain subgroups of $G$. In [17], M. Takesaki generalized this result to include covariance algebras (i.e., where $\alpha$ is trivial), and in [10], H. Leptin generalized the result to the twisted group algebra case with $T$ multiplicative. We give here an extension of this result to the general twisted group algebra case.

COROLlaRy 4.9. Let $A$ be a type I $C^{*}$-algebra, and suppose the orbit space $\hat{A} / G$ is countably separated. Then every factor representing pair $(\pi, U)$ of $(G, A ; T, \alpha)$ is induced (up to equivalence) by a representing pair $\left(\pi_{\gamma}, U_{\gamma}\right)$ of $\left(G_{\gamma}, A ; T, \alpha\right)$ for some point $\gamma \in \hat{A}$ such that $\pi_{\gamma}$ is a factor representation which is quasi-equivalent to a member of $\gamma$ and $G_{\gamma}$ is the stability group of $\gamma$.

Proof. We do not give a proof here. The proof of [17, Theorem 6.1] given by M. Takesaki may be modified to give a proof of this theorem. The proof requires many preliminary results and technical lemmas whose proofs follow, for the most part, those of Takesaki. The introduction of $\alpha$ presents many technical difficulties but does not drastically alter the techniques involved. Some of the needed results are presented in the material which precedes the corollary. For the person who is genuinely interested in seeing the details, see [1].

5. Primitive ideal spaces. This section is devoted to the study of the primitive ideal space of the twisted group algebra $L^{1}(G, A ; T, \alpha)$. The goal is to obtain the primitive ideal space of the twisted group algebra by inducing representations from stability groups. We generalize some of the results of Effros and Hahn in [6] to the general twisted group algebra case, and some to the more restricted case where the cocycle $\alpha$ is continuous. The major difficulty arises because the algebra $A$ is not commutative. When $A$ is commutative, the dual space of $A$ can be identified with the space of characters of $A$ (that is, complex-valued, nontrivial *-homomorphisms of $A$ ), so it is unnecessary to consider equivalence classes of representations of $A$. In the noncommutative case, however, equivalence classes must be considered. This results in the problem of having to choose the correct representative from the equivalence class and is one 
of the major reasons that Effros and Hahn's proofs break down in this setting. Another major obstacle in generalizing the results to twisted group algebras (even when $A$ is commutative) is that when $\alpha$ is present, the twisted product of two continuous functions with compact support need not be continuous. This is the reason that part of our results require continuity of $\alpha$.

We are now in position to prove the theorem which solves the first part of our problem. A group $G$ is said to be amenable if the left regular representation $U_{L}$ of $C^{*}(G)$ is faithful (that, has only zero in its kernel). Let $K(G)$ and $K(G, A)$ denote the continuous functions (complex-valued and $A$-valued, respectively) with compact support. It is well known that these spaces are dense in $L^{1}(G)$ and $L^{1}(G, A)$, respectively.

THEOREM 5.1. Let $A$ be type I. Let $G$ be amenable and act freely on $\hat{A}$. Let $L$ be an irreducible representation of $L^{1}(G, A ; T, \alpha)$ (where $(T, \alpha)$ is a smooth twisting pair) with corresponding representing pair $(\pi, U)$. The support of $\pi$ in $\hat{A}$ is an orbit closure, say $\overline{\left[\pi_{0}\right] G}$. In this case, $L$ is weakly contained in $I\left(\pi_{0}\right)$. (Hence, as representations of $C^{*}(G, A ; T, \alpha), \operatorname{ker} I\left(\pi_{0}\right) \subseteq \operatorname{ker} L$.)

Proof. From Lemma 4.4 and Corollary 4.8 , it suffices to show that $L$ is weakly contained in $L^{\otimes}$, where $L^{\otimes}$ is the integrated form of $\left(\pi \otimes I, U \otimes U_{L}\right)$, $U_{L}$ being the left regular representation of $G$.

By [7, Lemma 3.4] and [6, p. 66], there exists a net of continuous positive functionals $\left\{q_{i}\right\}$ on $K(G)$ such that $q_{i}(f)=\left(W_{i}(f) x_{i} \mid x_{i}\right)$ and $q_{i}(f) \rightarrow q(f)$ for every $f \in K(G)$, where $q$ is the positive functional on $K(G)$ corresponding to Haar measure on $G$ and $W_{i}$ is unitarily equivalent to a subrepresentation of the left regular representation of $C^{*}(G)$. Thus, $U_{i}$, the representation of $G$ corresponding to $W_{i}$, is equivalent to a subrepresentation of $U_{L}$. If $V_{i}$ sets up this equivalence then $I \otimes V_{i}$ sets up an equivalence between $L_{i}$, the integrated form of $\left(\pi \otimes I_{i}, U \otimes U_{i}\right)$, and a subrepresentation of $L^{\otimes}$. Hence $L_{i}$ is weakly contained in $L^{\otimes}$ for each $i$.

Now let $x$ and $y$ be arbitrary vectors in the Hilbert space of $L$. Then for all $f \in K(G, A)$,

$$
\begin{aligned}
\left(L_{i}(f)\left(x \otimes x_{i}\right) \mid y \otimes x_{i}\right) & =\int\left(\left[\pi(f(s)) U(s) \otimes U_{i}(s)\right]\left(\dot{x} \otimes x_{i}\right) \mid y \otimes x_{i}\right) d s \\
& =\int(\pi(f(s)) U(s) x \mid y)\left(U_{i}(s) x_{i} \mid x_{i}\right) d s .
\end{aligned}
$$

Let $g(s)=(\pi(f(s)) U(s) x \mid y)$. Then $g \in K(G)$, so

$$
\int g(s)\left(U_{i}(s) x_{i} \mid x_{i}\right) d s=q_{i}(g) \rightarrow q(g)=\int g(s) d s=(L(f) x \mid y) .
$$

Thus $L$ is weakly contained in $\left\{L_{i}\right\}$ and hence in $L^{\otimes}$. This completes the proof. We now investigate the second half of the problem; that is, with notations as 
in Theorem 5.1, we investigate the problem of determining whether $I\left(\pi_{0}\right)$ is weakly contained in $L$. For this, it has, unfortunately, been necessary to use the idea of induced representation in the sense of Effros and Hahn. This is the alternate definition of induced representation which we give in $\$ 3$.

Before stating the main theorem, we need the following result. If $L$ is a representation of $L^{1}(G, A ; T, \alpha)$, let $\bar{L}$ denote the corresponding representation of $C^{*}(G, A ; T, \alpha)$.

Proposition 5.2. Let $J$ be a closed ideal in $C^{*}(G, A ; T, \alpha)$, where $\alpha$ is continuous. Suppose there exist positive functionals $\left\{\phi_{i}\right\}$ and $\phi$ of $K(G, A ; T, \alpha)$ such that $J \subseteq \operatorname{kernel} \bar{L}\left(\phi_{i}\right)$ for each $i$ and $\phi_{i}(f) \rightarrow \phi(f)$ in $K(G, A ; T, \alpha)$. Then $J \subseteq \operatorname{kernel} \bar{L}(\phi)$.

Proof. With a few minor modifications, the portion of the proof of $[6$, Theorem 5.11] on p. 66 works in the more general setting.

THEOREM 5.3. Suppose $A$ is type I and $G$ acts freely on $\hat{A}$. Let $P$ be a primitive ideal of $C^{*}(G, A ; T, \alpha)$, where $\alpha$ is continuous. If there exists a representation $L$ of $L^{1}(G, A ; T, \alpha)$ with kernel $\bar{L}=P$ such that the corresponding representing pair $(\pi, U)$ has $U$ norm continuous, then $P \subseteq$ kernel $\bar{I}\left(\pi_{0}\right)$, where $\pi_{0}$ is an element in the support of $\pi$.

Proof. As pointed out in [17], we may assume without loss of generality that $\pi$ and the central system of imprimitivity have uniform multiplicity $n$; i.e., we may assume that $\pi=\int_{\hat{A}}^{\oplus} \pi_{\gamma} d \mu(\gamma)$ where $\pi_{\gamma}$ is a factor representation for almost every $\gamma$, and $H_{\pi}=L^{2}(\hat{A}, H, \mu)$. Furthermore, by [17, Theorem 4.1] and [1, Theorem 4.3], there exists a function $W$ mapping $\hat{A} \times G$ into the unitary operators on $H$ such that for almost every $\gamma$ in $\hat{A}$,

$$
(U(s) f)(\gamma)=\lambda(\gamma, s)^{1 / 2} W(\gamma, s) f(\gamma s)
$$

for all $f \in H_{\pi}$, where $\lambda$ is the $\lambda$-function corresponding to $\mu$.

Let $v$ be an element of norm one in the Hilbert space $H_{0}$ of $\pi_{0}$, where $\pi_{0}$ is any element in the support of $\pi$. Let $\phi(f)=\left(\pi_{0}(f(e)) v \mid v\right)$ for all $f \in K(G, A ; T, \alpha)$. We show that $\phi$ is the pointwise limit of positive functionals associated with $L$, and the theorem will follow from Proposition 5.2, since if $\psi$ is such a functional, then $L(\psi)$ is equivalent to a subrepresentation of $L$.

Given $f_{1}, \cdots, f_{m}$ in $K(G, A ; T, \alpha)$ and $\epsilon>0$, we show that there is a positive functional $p$ associated with $L$ which approximates $\phi$ to within $\epsilon$ on the $f_{i}$. Choose a compact set $C$ with support $f_{i} \subseteq C$ for all $i$. As $\pi$ and $U$ are both norm continuous, we may choose a compact neighborhood $N$ of $e$ in $G$ such that $\left\|\pi\left(f_{i}(s)\right) U(s)-\pi\left(f_{i}(e)\right)\right\|<\epsilon / 2$ for all $s$ in $N$. Let 
$C_{1}=C-N$. Since $C_{1}$ is compact and $G$ acts freely on $\hat{A}$, there exists a neighborhood $Q$ of $\left[\pi_{0}\right]$ such that $Q C_{1} \cap Q$ is empty. Let $R$ be a neighborhood of $\pi_{0}$ in the space of irreducible representations such that $\left|\left(\sigma\left(f_{i}(e)\right) v \mid v\right)-\left(\pi_{0}\left(f_{i}(e)\right) v \mid v\right)\right|<\epsilon / 2$ for every $\sigma$ in $R$. Let $S$ be the intersection of $Q$ with the image of $R$ in $\hat{A}$. Let $H_{0}^{\prime}$ be a Hilbert space such that $H=H_{0} \otimes H_{0}^{\prime}$, and let $I_{0}$ be the identity on $H_{0}^{\prime}$. Take $v_{1} \in H_{0}^{\prime}$ of norm one and form $w=v \otimes v_{1}$. Then for every $\sigma$ in $R$,

$$
\left|\left(\left(\sigma\left(f_{i}(e)\right) \otimes I_{0}\right) w \mid w\right)-\phi\left(f_{i}\right)\right|<\epsilon / 2 .
$$

Since $R$ is a Polish space, there exists a measurable cross-section $\left[\sigma_{\gamma}^{\prime}\right]$ in $R$. Let $\sigma_{\gamma}=\sigma_{\gamma}^{\prime} \otimes I_{0}$. For almost every $\gamma, \pi_{\gamma}$ and $\sigma_{\gamma}$ are quasi-equivalent and have the same multiplicity and hence, since $A$ is type $\mathrm{I}$, are equivalent. By [5, Proposition 8.2.3], there exists a unitary $V=\int_{S}^{\oplus} V(\gamma) d \mu(\gamma)$ such that $V(\gamma) \sigma_{\gamma}(a) V(\gamma)^{*}=\pi_{\gamma}(a)$ for every $a$ in $A$ and almost every $\gamma$ in $S$. Define $x(\gamma)=V(\gamma) w$ for every $\gamma$ in $S$ and $x(\gamma)=0$ for all other $\gamma$ in $\hat{A}$. Let $x=\int_{\hat{A}}^{\oplus} x(\gamma) d \mu(\gamma)$. We have that $\left|\left(\pi_{\gamma}\left(f_{i}(e)\right) x(\gamma) \mid x(\gamma)\right)-\phi\left(f_{i}\right)\right|<\epsilon / 2$ for almost every $\gamma$ in $S$.

Choose $B \in L^{\infty}(A, \mu)$ such that $B \geqslant 0, \int B(\gamma)^{2} d \mu(\gamma)=1$, and $B$ vanishes outside of $S$. Such a $B$ exists since $\left[\pi_{0}\right] \in$ support of $\mu$ and $\left[\pi_{0}\right] \in S$. Define $E=B /(d s(N))^{1 / 2}$ and $p(f)=(L(f) E x \mid E x)$. We show that $p$ has the desired properties. Note that

$$
\begin{aligned}
p\left(f_{i}\right) & =\int_{C}\left(\pi\left(f_{i}(s)\right) U(s) E x \mid E x\right) d s \\
& =\int_{C} \int_{\hat{A}}\left(\pi_{\gamma}\left(f_{i}(s)\right) \lambda(\gamma, s)^{1 / 2} W(\gamma, s) E(\gamma s) x(\gamma s) \mid E(\gamma) x(\gamma)\right) d \mu(\gamma) d s \\
& =\int_{N}\left(\pi\left(f_{i}(s)\right) U(s) E x \mid E x\right) d s .
\end{aligned}
$$

The last equality follows from the fact that $x(\gamma)=0$ if $\gamma \notin S$ and if $\gamma \in S$ and $s \in C_{1}$, then $s$ is not in $s$, so $x(\gamma s)=0$. Let

$$
F_{1}=\int_{N}\left(\pi\left(f_{i}(e)\right) E x \mid E x\right) d s=\left(\pi\left(f_{i}(e)\right) B x \mid B x\right) .
$$

We have

$$
\begin{aligned}
\left|p\left(f_{i}\right)-F_{i}\right| & =\left|\int_{N}\left(\left[\pi\left(f_{i}(s)\right) U(s)-\pi\left(f_{i}(e)\right)\right] E x \mid E x\right) d s\right| \\
& \leqslant \int_{N}\left\|\pi\left(f_{i}(s)\right) U(s)-\pi\left(f_{i}(e)\right)\right\|(E x \mid E x) d s \\
& <(\epsilon / 2) \int_{N}(E x \mid E x) d s=(\epsilon / 2)(B x \mid B x) \\
& =(\epsilon / 2) \int_{S} B(\gamma)^{2}(x(\gamma) \mid x(\gamma)) d \mu(\gamma)=(\epsilon / 2) \int_{S} B(\gamma)^{2} d \mu(\gamma)=\epsilon / 2
\end{aligned}
$$

Furthermore 


$$
\begin{aligned}
\left|F_{i}-\phi\left(f_{i}\right)\right| & =\left|\left(\pi\left(f_{i}(e)\right) B x \mid B x\right)-\left(\pi_{0}\left(f_{i}(e)\right) v \mid v\right)\right| \\
& =\left|\int_{S}\left(\pi_{\gamma}\left(f_{i}(e)\right) B(\gamma) x(\gamma) \mid B(\gamma) x(\gamma)\right) d \mu(\gamma)-\int_{S} B(\gamma)^{2}\left(\pi_{0}\left(f_{i}(e)\right) v \mid v\right) d \mu(\gamma)\right|^{\mid} \\
& =\left|\int_{S}\left[\left(\pi_{\gamma}\left(f_{i}(e)\right) x(\gamma) \mid x(\gamma)\right)-\left(\pi_{0}\left(f_{i}(e)\right) v \mid v\right)\right] B(\gamma)^{2} d \mu(\gamma)\right| \\
& <(\epsilon / 2) \int_{S} B(\gamma)^{2} d \mu(\gamma)=\epsilon / 2 .
\end{aligned}
$$

Thus $\left|p\left(f_{i}\right)-\phi\left(f_{i}\right)\right|<\epsilon$. This concludes the proof.

As a consequence of Theorems 5.1 and 5.3 we get the following corollary, which includes as a special case a result obtained by Zeller-Meier in [18].

COROLlary 5.4. If $G$ is discrete, amenable, and acts freely on $\hat{A}$, with $A$ type $\mathrm{I}$, then there exists a one-to-one correspondence between orbit closures in $\hat{A}$ and the primitive ideal space of $C^{*}(G, A ; T, \alpha)$.

6. Conclusion. The problem of obtaining the dual space of the twisted group algebra by inducing has been solved in the general setting if $A$ is type $I$ and $G$ acts smoothly on $A$. The problem of obtaining the primitive ideal space of $C^{*}(G, A ; T, \alpha)$ has been solved to a certain degree when the pair $(T, \alpha)$ is smooth. Part of the solution, however, required continuity of $\alpha$. It appears that the same techniques can be used even if $\alpha$ is not continuous by considering equivalent twisting pairs as was done in [3], but we have thus far been unable to obtain the desired results in this way.

In [7], E. Gootman was able to relax the conditions of free action and norm continuity required in Theorems 5.1 and 5.3 (for the transformation group case). We see no immediate way to carry through Gootman's generalization to the twisted group algebra case. The problem of generalizing free action causes complications since if $\alpha$ is present or if $A$ is not commutative, a $\sigma$-representation of $G$ is required for the inducing so the one-dimensional identity representation cannot be used to induce and it is not at all clear which $\sigma$-representation should be used. To relax the norm continuity condition, Gootman assumed the existence of a uniform eigenvector for all $U(s)$. We encounter difficulty here because we have to use equivalence classes of representations of $A$ and hence it is difficult to use a fixed vector throughout, as one may see in our proof of Theorem 5.3.

\section{REFERENCES}

1. O. L. Britton, Dual spaces and primitive ideal spaces of twisted group algebras, Doctoral Dissertation, Drexel University, Philadelphia, Pa., 1972.

2. R. C. Busby, Double centralizers and extensions of $C^{*}$-algebras, Trans. Amer. Math. Soc. 132 (1968), 79-99. MR 37 \#770.

3. - On a theorem of Fell, Proc. Amer. Math. Soc. 30 (1971), 133-140. MR 44 \#814.

4. R. C. Busby and H. A. Smith, Representations of twisted group algebras, Trans. Amer. Math. Soc. 149 (1970), 503-537. MR 41 \#9013. 
5. J. Dixmier, Les $C^{*}$-algèbres et leurs représentations, Cahiers Scientifiques, fasc. 29 , Gauthier-Villars, Paris, 1964. MR 30 \#1404.

6. E. G. Effros and F. Hahn, Locally compact transformation groups and $C^{*}$-algebras, Mem. Amer. Math. Soc. No. 75 (1967). MR 37 \#285.

7. E. C. Gootman, Primitive ideals of $C^{*}$-algebras associated with transformation groups, Trans. Amer. Math. Soc. 170 (1972), 97-108. MR 46 \#1961.

8. E. Hille and R. S. Phillips, Functional analysis and semi-groups, rev. ed., Amer. Math. Soc. Colloq. Publ., vol. 31, Amer. Math. Soc., Providence, R. I., 1957. MR 19, 664.

9. B. E. Johnson, An introduction to the theory of centralizers, Proc. London Math. Soc. (3) 14 (1964), 299-320. MR 28 \#2450.

10. H. Leptin, Darstellungen Verallgemeinerte $L^{1}$-Algebren. II (to appear).

11. - Verallgemeinerte $L^{1}$-Algebren, Math. Ann. 159 (1965), 51-76. MR 39 \#1909.

12. - Verallgemeinerte $L^{1}-$ Algebren and projektive Darstellungen lokal kompakter Gruppen. I, Invent. Math. 3 (1967), 257-281. MR 37 \#5328.

13. - Verallgemeinerte $L^{1}$-Algebren and projective Darstellungen lokal kompakter Gruppen. II, Invent. Math. 4 (1967), 68-86. MR 37 \#5328.

14. G. Mackey, Induced representations of locally compact groups. I, Ann. of Math. (2) 55 (1952), 101-139. MR 13, 434.

15. - Unitary representations of group extensions. I, Acta Math. 99 (1958), 265-311. MR 20 \#4789.

16. M. A. Naĭmark, Normed rings, 2nd rev. ed., "Nauka", Moscow, 1968; English transl., Wolters-Noordhoff, Groningen, 1970.

17. M. Takesaki, Covariant representations of $C^{*}$-algebras and their locally compact automorphism groups, Acta Math. 119 (1967), 273-303. MR 37 \#774.

18. G. Zeller-Meier, Produits croisés dans une $C^{*}$-algèbre par un groupe d'automor phismes, C. R. Acad. Sci. Paris Sér. A-B 263 (1966), A20-A23. MR 33 \#7877.

DEPARTMENT OF MATHEMATICS, UNIVERSITY OF MISSISSIPPI, UNIVERSITY, MISSISSIPPI 38677 